University of New Hampshire

University of New Hampshire Scholars' Repository

8-1987

\title{
EELGRASS WASTING DISEASE: CAUSE AND RECURRENCE OF A MARINE EPIDEMIC
}

\author{
Frederick T. Short \\ University of New Hampshire, fred.short@unh.edu \\ Lisa K. Muehlstein \\ David Porter
}

Follow this and additional works at: https://scholars.unh.edu/jel

\section{Comments}

This is an article published by University of Chicago Press in Biological Bulletin in 1987, available online:

https://doi.org/10.2307/1541701

\section{Recommended Citation}

Short, F.T., L.K. Muelstein, and D. Porter. 1987. Eelgrass Wasting Disease: cause and recurrence of a marine epidemic. Biological Bulletin 173:557-562.

This Article is brought to you for free and open access by the Institute for the Study of Earth, Oceans, and Space (EOS) at University of New Hampshire Scholars' Repository. It has been accepted for inclusion in Jackson Estuarine Laboratory by an authorized administrator of University of New Hampshire Scholars' Repository. For more information, please contact Scholarly.Communication@unh.edu. 


\section{ABSTRACTS OF PAPERS PRESENTED AT THE MARINE BIOLOGICAL LABORATORY: NORTHEASTERN REGIONAL CONFERENCE ON DEVELOPMENTAL BIOLOGY 24-27 SEPTEMBER, 1987}

The conference was organized by Lorraine Gudas (Dana-Farber Cancer Institute and Harvard Medical School) and Greenfield Sluder (Worcester Foundation for Experimental Biology). Support for graduate student participation was provided by the $\mathrm{Na}$ tional Science Foundation (DCB-8713713).

\section{ABSTRACTS}

In vivo properties of primary cilia in cultured kidney epithelial cells. S. S. BOWSER, K. E. ROTH, AND C. L. RIEDER (Wadsworth Ctr. Labs. \& Res., NY State Dept. Health, Albany, NY).

A single primary $\left(1^{\circ}\right)$ cilium is generated from the parent centriole of the centrosome in most embryonic and terminally differentiated cell types. $1^{\circ}$ cilia are oriented perpendicular to the dorsal cell surface, which makes them difficult to observe by conventional LM preparative methods. As a result, their properties and function remain unknown. To overcome this restriction, we grow kidney epithelial cell monolayers on plastic films. A side view of the monolayer is obtained by folding the film, cell-side out, and sandwiching it in a simple coverslip chamber. Along the folded edge, $1^{\circ}$ cilia are perpendicular to the optical axis and readily imaged by real-time or time-lapse video-LM. $1^{\circ}$ cilia appear as rigid, $0.25 \mu \mathrm{m}$-diameter rods and their average length is characteristic for each kidney cell line examined. $1^{\circ}$ cilia show no beating motions but passively bend in response to flow. $1^{\circ}$ cilia frequently possess one or more varicosities or swellings along the ciliary shaft. Time-lapse video LM reveals that these varicosities move bidirectionally between the ciliary base and tip. Same-cell correlative LM and high-voltage EM indicates that these varicosities are delimited by the ciliary membrane and contain a granular cytoplasmic matrix devoid of vesicles or other organelles. Our finding that cytoplasmic transport occurs along $1^{\circ}$ cilia suggests they may serve as a conduit for exchange between the extracellular milieu and the nucleus.

Time-lapse photographic study of neural tube closure defects in the chick. K. T. BuSH, R. G. NAGELE, AND H. LEE (Department of Biology, Rutgers University, Camden, NJ and Department of Pediatrics, University of Medicine \& Dentistry of New Jersey-School of Osteopathic Medicine, Camden, NJ).

A new method was described whereby early chick embryos were explanted using a modified New's technique [in which (1) avian Ringer's solution replaced Pannett-Compton saline and (2) the vitelline membrane with an adherent blastoderm was placed over the glass ring] and photographed with a Nikon Diaphot inverted microscope equipped with both phase-contrast optics and photomicrographic accessories maintained in an incubator. This method permits for the first time recording of sequential changes in the morphology of early chick embryos. Specifically, 4-somite stage embryos were explanted, grown in nutrient medium with or without a teratologic dose of xylocaine (lidocaine $\mathrm{HCl}$, Elkins-Sinn), and were photographed at 2-3 hour intervals. We found, among other things, that a characteristic neural tube closure defect often seen in the brain region of xylocaine $(200 \mu \mathrm{g} / \mathrm{ml})$-treated chick embryos was consequence of failure of the neural tube to withstand the tension generated by the rapidly expanding cephalic region which occurred when the corresponding controls had advanced to 11 -somite stage.

Interspecific comparisons of the period gene. H. V. COlOT, G. PETERSEN, Q. Yu, D. WHEELER, J. C. HALL, AND M. ROSBASH (Department of Biology, Brandeis University, Waltham, MA).

We have cloned and sequenced substantial portions of the period (per) gene from Drosophila pseudoobscura, $D$. virilis, and $D$. simulans as part of our continuing investigation into the function of the per gene 
in circadian and ultradian rhythms. The $D$. pseudoobscura gene has been examined in the most detail and compared with the well-characterized per gene of $D$. melanogaster. The locomotor activity of $D$. pseudoobscura differs substantially in period length and strength, as well as overall pattern, from that of $D$. melanogaster. DNA sequence comparison between these two species shows blocks of diverged coding sequence interspersed with blocks of conserved coding sequence. Surprisingly, the TG-repeat region of $D$. melanogaster is replaced in $D$. pseudoobscura by a longer, totally different repeat that is preceded by only a few TG and SG pairs. (Note that deletion of most of the TG region of D. melanogaster affects the speciesspecific courtship song rhythm but not the circadian rhythm.) Therefore, the TG repeat is probably not a series of (obligatory) glycosaminoglycan attachment sites but may confer species-specific properties on the protein. Experiments involving cross-species transformation of the per gene have been initiated.

\section{Pattern formation with Fusarium illustrates a principle for generation of cell pattern. J. \\ DAS AND H. BUSSE (Biochem. Inst. Med. Fak., Univers. Kiel, GFR and Harvard Medical School, Dept. Physiology \& Biophys., Boston, MA).}

The fungus Fusarium usually grows by extending its mycelium outwards. If hyphae try to grow into a region already occupied by the mycelium of a neighboring fungus, then either of the two following processes can occur: (1) the hyphae do not recognize each other and intermingle; or (2) the hyphae recognize that the neighboring region is already occupied and stop growing.

In the second case, the pattern formed in the plane of growth can be understood in terms of a specific distance measure. A distance measure is a mathematical function which allows an object (a hypha in our case) to determine its distance relative to other objects in the neighborhood. If the distance measure reaches a preset limit a decision is made. In our example: hyphae stop to grow. That distance measures can produce spatial pattern is illustrated for Fusarium. It is likely that patterns in differentiating animal tissue can also be described by distance measures.

Integrin structure, function, and developmental expression. D. W. DESIMONE, M. A. STEPP, R. PATEL, E. MARCANTONIO, AND R. O. HYNES (Center for Cancer Research, M.I.T., Cambridge, MA).

The integrins are a family of cell-surface receptor complexes that participate in a variety of cell-cell and cell-extracellular matrix interactions, which are involved in morphogenesis, cell migration, hemostasis, oncogenesis, and immune function. Integrins exist as heterodimers made up of distinct $\alpha$ and $\beta$ subunits. We have isolated cDNA clones of integrin subunits from several species. Comparisons among the cDNA sequences of $\beta$ subunits from Xenopus laevis, chicken, mouse, and human reveal that the integrin family can be organized into at least three classes. We estimate that the three classes of $\beta$ subunits diverged from a common ancestral gene at an early stage of metazoan evolution. Functional heterogeneity within integrin classes is probably conferred by a large number of distinct, yet structurally related, $\alpha$ subunits. The structural relatedness of the integrin receptors will be discussed in view of results obtained by Northern and Southern blot hybridization analyses, cDNA sequencing, and immunologic cross-reactivity. Similar approaches are being used to determine the developmental expression and functional significance of the integrins during amphibian embryogenesis.

Identification of a calcium-calmodulin dependent protein kinase associated with the sea urchin mitotic apparatus. J. H. DINSMORE AND R. D. SLOBODA (Dept. of Biological Sciences, Dartmouth College, Hanover, NH 03755).

Mitotic apparatuses (MAs) isolated from the sea urchin, Lytechinus pictus, were assayed for protein kinase activity; phosphorylation of endogenous MA substrates was determined by SDS-polyacrylamide gel electrophoresis and autoradiography. MAs were isolated by the Salmon (Methods Cell Biol. 25: 71-102, [1982]) detergent lysis method with the following modifications: embryos were treated with $3 \%$ hexylene glycol for 3 min prior to lysis; $50 \mu M$ GTP and protease inhibitors were included in the lysis buffer, and MAs were washed two times in $50 \mathrm{mM}$ PIPES, pH 6.9 plus $10 \mu M$ taxol to obtain the final MA preparation. Isolated MAs were incubated with $\left[\gamma^{32} \mathrm{P}\right] \mathrm{ATP}, \mathrm{Mg}^{2+}$, and one of the following; $\mathrm{CAMP} ; \mathrm{CGMP}^{2} \mathrm{Ca}^{2+} ; \mathrm{cal}^{-}$ modulin; $\mathrm{Ca}^{2+}$ and calmodulin; $\mathrm{Ca}^{2+}$, calmodulin and calmidizolium. Specific phosphorylation of only a single protein of $\mathrm{Mr} 62 \mathrm{kD}$ occurred when both $\mathrm{Ca}^{2+}$ and calmodulin were present. Incubation of intact, birefringent MAs with $40 \mu M$ ATP plus $0.3 \mu M$ calmodulin resulted in an increased rate and extent of depolymerization of MA microtubules in response to micromolar $\mathrm{Ca}^{2+}$. In the absence of either ATP or calmodulin this effect of $\mathrm{Ca}^{2+}$ on microtubule stability was not observed. Finally, in vivo phosphorylation experiments showed that protein phosphorylation in the embryo rose and fell with the cell cycle, with peak 
levels of phosphorylation occurring at the metaphase-anaphase transition. The results suggest that specific $\mathrm{Ca}^{2+}$-calmodulin dependent protein phosphorylation affects MA stability, and may be important in controlling the metaphase-anaphase transition in vivo. [Supported by NSF BNS85-03597 to RDS and a R. M. Cramer Graduate Fellowship and Sigma Xi Grant-in-Aid to JHD]

Inducible c-myc overexpression and F9 teratocarcinoma stem cell differentiation. C. A. DionNe AND L. J. GudAs (Division of Cancer Genetics, Dana-Farber Cancer Institute and Pharmacology Department, Harvard Medical School, Boston, MA).

The retinoic acid-induced terminal differentiation of $\mathrm{F} 9$ teratocarcinoma stem cells is accompanied by a very early and rapid decrease in c-myc proto-oncogene expression. We have isolated several F9 cell lines which have stably integrated a tightly regulated, highly inducible c-myc expression vector. The morphological and biochemical differentiation of these transfected cell lines was similar to that of F9 wild type cells despite continuous induced c-myc overexpression throughout the differentiation period. In addition, c-myc overexpression did not prevent the decreased rate of cellular proliferation in response to retinoic acid. Our results argue that the previously reported dominant effects of c-myc on differentiation and cellular proliferation are not observed in embryonic stem cells such as F9 teratocarcinoma stem cells.

Nucleoprotein complexes that regulate gene expression in adipocyte differentiation: direct participation of $c$-fos. ROBERT J. DISTEL, HYO-SUNG RO, BARRY S. ROSEN, DOUglas L. GROVES, AND BRUCE M. SPIEGELMAN (Dana-Farber Cancer Institute and Department of Pharmacology, Harvard Medical School, Boston, MA).

Adipocyte differentiation is accompanied by the transcriptional activation of many new genes, including a putative lipid binding protein termed adipocyte $\mathrm{P} 2(\mathrm{aP} 2)$. The aP2 gene contains a regulatory element (FSE2) 124 bases $5^{\prime}$ to its start of transcription. This DNA sequence binds nuclear factors in a sequencespecific fashion as determined by its altered mobility in gel retardation assays. Deletion analysis of promotor-linked transfection assays and competition of these constructions in cells with synthetic FSE2 elements suggests that trans-acting factors bind in this region and act as negative regulators of aP2 gene activity in preadipocytes. c-fos appears to participate directly in this nucleoprotein complex, as demonstrated by the ability of antibodies to c-fos to disrupt specific binding of factors and the FSE2 sequence but not to factorbinding sequences from several other genes. Antibodies to c-fos specifically immunoprecipitate protein complexes covalently bound to FSE2 DNA via UV cross-linking.

\section{Intracellular patterning and the problem of assembly. GARY W. GRIMES (Department} of Biology, Hofstra University, Hempstead, NY 11550).

Ciliated protozoa typically are described on the basis of their highly asymmetric pattern of the ciliature. However, we have microsurgically induced a ciliate possessing bilateral symmetry which is stably inherited during asexual (and presumably sexual) reproduction. Because these bilaterally symmetrical cells are essentially "Siamese Twins" fused side-by-side, with one half the mirror image of the other, one would predict that the asymmetry of the individual ciliary structures would correspondingly be mirror-imaged. However, they are not. Rather, they are assembled typically (i.e., not mirror-imaged) and patterned in a mirrorimaged manner, or they are assembled in an inverted fashion but outwardly organized in a mirror-imaged pattern in order to correspond to the global asymmetry of the mirror-imaged half of the cell. These data suggest the presence of a global patterning mechanism imposed on the cortex of these ciliates to which the individual elements of the ciliature must respond during their assembly. Constraints on the way in which the ciliature can be assembled thus determines whether or not they are assembled in a "typical" or inverted manner. The general principles of "directed assembly" and "directed patterning" are emphasized by these studies. Supported by Research Grants from the NSF and Hofstra University.

Cell surface reorganization in the fertilized egg of the zebrafish. N. H. HART AND J. S. WOLENSKI (Department of Biological Sciences, Rutgers University, New Brunswick, NJ).

The time-course of surface reorganizational events and their sensitivity to microfilament inhibitors in the fertilized egg of the zebrafish (Brachydanio) were studied with light and electron microscopy. A single 
sperm binds to a predetermined site on the egg oolemma within $5 \mathrm{~s}$ of insemination. A distinct fertilization cone formed at this site between 45-60 s. Complete incorporation of sperm head, midpiece, and a portion of the flagellum occurred between 60 and $120 \mathrm{~s}$. The second polar body formed by 4 min near the site of gamete fusion. Cortical granule exocytosis, initiated beyond the site of gamete union by $30 \mathrm{~s}$, was completed by 4-5 min. The reaction in the vicinity of the fertilizing sperm was visible by $60 \mathrm{~s}$. Freeze fracture analysis showed higher IMP density on the P leaflet of the egg plasma membrane than on the $P$ face of the cortical granule membrane. These differences persisted after cortical granule breakdown, suggesting that the two membrane domains do not mix rapidly. Cortical granule exocytosis and fertilization cone formation were unaffected by either cytochalasin $B$ or $D(10 \mu \mathrm{g} / \mathrm{ml})$ treatment. Sperm incorporation was inhibited by either $10 \mu \mathrm{g} / \mathrm{ml} \mathrm{CB}$ or $50 \mu \mathrm{g} / \mathrm{ml} \mathrm{CD}$. Sperm entry into the egg appears to require actin polymerization.

Molecular studies of biological rhythms in Drosophila. F. R. JACKSON AND K. J. ELLIOTT (Worcester Foundation for Experimental Biology, Shrewsbury, MA).

The fruitfly Drosophila is an excellent model system for genetic studies of biological clocks. As a consequence, several Drosophila genes have been identified that determine the properties of biological oscillators. One of these genes, period (per), encodes a proteoglycan-like glycoprotein whose function is currently being investigated in several laboratories. We have recently employed per-gene probes to identify and isolate segments of Drosophila genomic DNA containing related sequences. One of these 'per-homologous' clones appears to encode a proteoglycan which contains Thr-Gly repeats similar to those seen in the per protein. Experiments are underway to determine the relevance of this 'per-homologous' gene for the development and/or maintenance of circadian rhythms.

Another Drosophila clock gene, Andante, maps to chromosomal region $10 \mathrm{E}$ and lengthens periods of circadian and ultradian rhythms. We have cloned DNA sequences from the $10 \mathrm{E}$ region, and initiated a chromosomal walk towards the And locus. Chromosomal breakpoints which flank the And gene are currently being localized in cloned sequences to define the physical limits of the locus.

Isolation and characterization of an $m R N A$ sequence (ERA-1) exhibiting a rapid and protein synthesis independent induction during the retinoic acid-induced differentiation of teratocarcinoma stem cells. G. J. LAROSA AND L. J. GUDAS (Program on Cell and Devel. Biology, Harvard Medical School and Dana-Farber Cancer Institute, Boston, MA).

Vitamin A and its derivatives (retinoids) exhibit profound effects on the proliferation and differentiation of many cell types. F9 teratocarcinoma stem cells, which differentiate into non-tumorigenic primative endoderm cells in response to retinoic acid (RA), serve as an excellent in vitro model for molecular studies of cellular differentiation and the mechanism by which RA can set this complex process into motion. In order to begin to analyze events which occur rapidly after RA treatment of F9 cells, we have constructed a cDNA library from RNA of F9 cells treated for $8 \mathrm{~h}$ with RA. By screening with a cDNA probe enriched for RA-induced sequences by subtractive solution hybridization, we have been able to isolate a cDNA clone for an RNA which exhibits the properties of a primary target of RA action. This gene, Early Retinoic Acid-1 (ERA-1), encodes a $2.2 \mathrm{~kb}$ polyadenylated RNA which is rapidly induced by RA, in a dose-dependent manner, both in the absence or presence of protein synthesis inhibitors. The isolation of this gene sequence demonstrates that RA can influence gene expression very rapidly and also provides us with an important tool to further analyze the initial intracellular action of RA in this F9 differentiation model system.

Cellular interactions during amphibian gastrulation. J. LEBLANC (The College of Staten Island, CUNY, NY), M. YODER AND I. BRICK (New York University, NY).

Epithelial movement during amphibian gastrulation is responsible for much of the reorganization that establishes the primitive body plan. If cells are to form an epithelium and then exhibit epithelial translocations, sufficient intercellular cohesion, adhesions along their adjacent edges, would be required to maintain integrity. Cellular interactions at the dorsal and lateral lip areas of the blastopore in Rana pipiens embryos at various stages during gastrulation have been investigated by TEM.

Areas of unspecialized apposition and desmosomes were observed between adjoining cell surfaces (1) of lip cells lining the blastoporal groove, (2) of lip cells at the surface of the groove and underlying lip cells, (3) of cells of the yolk plug lining the blastoporal groove, (4) of yolk plug cells at the surface of the groove and underlying yolk plug cells, and (5) of lip and yolk plug cells. The cellular arrangements and intercellular 
junctions suggest that the layer of lip cells and the layer of yolk plug cells that line the blastoporal groove are both an epithelium.

Primordial germ cells of day 5 chick embryos. B. Y. LEE, K. T. BUSH, R. G. NAGELE, AND H. LEE (Department of Pediatrics, University of Medicine \& Dentistry of New Jersey-School of Osteopathic Medicine, Camden, NJ and Department of Biology, Rutgers University, Camden, NJ).

Primordial germ cells (PGCs) of the chick are initially identifiable with certainty in the germinal crescent area of the definitive streak stage embryo. As embryonic development proceeds, most PGCs migrate to the area vasculosa, enter blood vessels, and then reach the gonads. By day 5 of development, nearly all PGCs, which will eventually settle in the gonads, are already in the gonads. These PGCs are distinguishable from somatic (germinal epithelial) cells in that they have well-defined cell boundaries, numerous periodic acid Schiff-positive granules, prominent lipid droplets, ring-shaped Golgi complex, and fragmented nucleoli. They appear to relocate themselves in an ameboid fashion within the gonads. Of the several methods used for isolating PGCs from the gonads, treatment with $0.2 \%$ collagenase- $0.1 \%$ trypsin inhibitor in calcium- and magnesium-free Hanks' balanced salt solution followed by the discontinuous Percoll density-gradient centrifugation was found to be most effective. Isolated PGCs, when grown in vitro, retained both their distinguishing features and the ability to migrate actively.

Leukemic viral expression with induced changes in hematopoietic microenvironment. G. P. LeONARdi, M. MaNThos, J. LoBue, D. ORlic, AND J. Mitra (Dep't of Biology, New York Univ., New York, NY and Dep't of Anatomy, N. Y. Medical College, Valhalla, NY).

A transplantable, granulocytic leukemia has been established from BALB/c mice inoculated with a variant of Rauscher Leukemia Virus that normally induces severe derangement of erythropoiesis (RLVA; LoBue et al., 1972). Six weeks before, and following inoculation of this erythroblastic virus, these mice were kept at elevated red cell volumes by injection of $75 \%$ packed red cells (i.e., hypertransfusion) every 7 days. Such hypertransfusion in mice eliminates red cell formation and alters the microenvironment making it more suitable for granulopoiesis. Development of the "typical" RLV-A pathogenesis resulting in erythroblastosis and fatal anemia was not observed in these animals. Instead, the appearance of massive numbers of leukocytic elements including transplantable monomyelocytic leukemic cells was seen in four of the six animals by 18-40 weeks post-viral inoculation. Hypertransfused control animals did not exhibit these reactions. Hypertransfusion eliminates production of red cells and their precursors, and modifies bone marrow stromal cells thus changing the microenvironment (Brookoff and Weiss, 1982). Sustained hypertransfusion by (1) eliminating virally susceptable erythroid cells or (2) modifying the hematopoietic microenvironment or (3) as a consequence of both resulted in the expression of a different viral oncogenic expression. The authors acknowledge the generous financial support of Dr. and Mrs. P. C. Chan for these studies.

Interleukin-2 (IL-2) distribution in adult newts (Notophthalmus viridescens) during regeneration and following non-amputational wounding. M. F. LOMBARD ${ }^{1}$ AND R. E. SiCARD ${ }^{2}$ ('Department of Biology, Regis College, Weston, MA; and ${ }^{2}$ Department of Pediatrics, Rhode Island Hospital, Providence, RI).

A role for the immune system in epimorphic regeneration has been proposed, but not proved. Accordingly, $\left[{ }^{3} \mathrm{H}\right]-\mathrm{IL}-2$ (a potent lymphokine that modulates immune response through its actions on macrophages and lymphocytes) was used to explore changes in distribution and availability of IL-2 responsive cells during regeneration and after non-amputational wounding. IL-2 content was reduced in major hemopoietic organs 2-8 days after trauma. Progressive increases, to control levels, occurred in livers of both groups; however, IL-2 content remained depressed in spleens of regenerating, but not "wounded," animals between 15-21 days post-trauma. During this same period, IL-2 content was reduced peripherally, as reflected by decreased content in non-traumatized forearms. Initially, IL-2 content of non-amputational wound sites was low, but progressively increased from 8-21 days post-trauma. In contrast, IL-2 content of stumps and blastemas decreased between 2-21 days postamputation. The apparent pattern of availability and distribution of IL-2 responsive cells during regeneration differed from that seen during repair of nonamputational wounds. These data are consistent with immunological influence on regeneration and suggest that further exploitation of IL-2 as a probe might help elucidate that role. 
Sea urchin H2A.F/Z: an unusually conserved H2A variant gene. ROBERT MCISAAC, Heidi Miller, Carol A. BrenNer, CATHERINe Nocente-MCGrath, SUSAN FRANCIS, AND SUSAN G. ERNST (Department of Biology, Tufts University, Medford, MA).

Sea urchin development is distinct in its sequential and overlapping use of multiple histone classes. In addition, variants have been isolated which are differentiation specific. We have identified a cDNA clone that encodes an H2A variant histone gene which is distinct from any class of sea urchin histones. The coding region is $74 \%$ homologous to the chicken H2A.F gene and a remarkable $97 \%$ conservation exists when comparing the putative amino acid sequences. Developmental expression does not coincide with any embryonic histone class and the message has been found in all adult tissues examined. The $1.7 \mathrm{~kb}$ transcript contains an unusually long $3^{\prime}$ untranslated region and binds to oligo dt cellulose. In contrast to the early gene set, this sequence is present at only a few copies per haploid genome. Both the sea urchin and the chicken gene encode a protein related to the replication-independent protein H2A.Z found in vertebrates. This protein is present at a level of $1-10 \%$ of the total H2A. Its low abundance, highly conserved nature, and broad phylogenetic distribution all suggest a unique role for this protein in chromatin organization.

This work was supported by a Biomedical Research Grant and Tufts University Faculty Research Award to Susan G. Ernst.

Luminescence proteins exhibit circadian rhythms but their mRNAs do not. D. MORSE, P. MILOS, E. ROUX, AND J. W. HASTINGS (Biological Labs, Harvard University, Cambridge, MA 02138).

At least three components of the bioluminescent system in Gonyaulax polyedra are under circadian regulation: the substrate (luciferin), the luciferin binding protein (LPB), and the enzyme luciferase. LPB cDNA was isolated by immunological screening from a cDNA library subcloned into an expression vector. Its identity was confirmed by in vitro translation of hybrid selected mRNA. Northern hybridization to mRNAs isolated at 8 different time points spanning a 24-hour period showed that the amounts of the LBP mRNA were invariant with time. A putative luciferase cDNA has also been isolated and experiments similarly indicate constant levels of the corresponding mRNAs. In a complementary approach, in vitro translation of the mRNAs used in the Northern experiments showed equal synthesis of LBP at all time points. These results indicate that circadian control of the amount of binding protein is exerted at the translational rather than the transcriptional level.

The guinea pig sperm surface protein $P H-20$ is anchored in the membrane by a phosphatidylinositol lipid. B. PHelPS, P. PRIMAKOFF, D. E. KoPPEL, AND D. G. MYLES. (Dept. of Physiology, University of Connecticut Health Center, Farmington, CT).

PH-20 is an integral protein of the guinea pig sperm plasma membrane, for which there is evidence for a role in sperm-zona pellucida binding [Primakoff et al., J. Cell Biol. 101: 2239 (1985)]. PH-20 is uniformly distributed over the entire cell surface of testicular sperm, but is localized to the posterior head region of distal cauda epididymal sperm. Following the exocytotic acrosome reaction the plasma membrane becomes contiguous with the inner acrosomal membrane and PH-20 migrates to the anterior head region [Myles and Primakoff, J. Cell Biol. 99: 1634 (1984)]. Measurement of the diffusion coefficient of PH-20 using the technique of fluorescence recovery after photobleaching (FRAP), show that PH-20 diffusion on testicular sperm is highly restricted within the plane of the bilayer, while PH-20 on acrosomereacted sperm is freely diffusing. At the intermediate stage, acrosome-intact cauda epididymal sperm, the protein diffuses at a rate between these two extremes; it is mobile but not freely diffusing. Exposure of testicular sperm, acrosome-intact, and acrosome-reacted epididymal sperm to a phosphatidylinositol-specific phospholipase $\mathrm{C}$ results in the release of PH-20 from the cell surface. Thus, a cell that is no longer capable of protein synthesis is able to modulate, during its differentiation, both the surface distribution and the rate of diffusion of an integral membrane protein that is anchored in the bilayer by a lipid. Supported by GM23585 to D.E.K. and NIH HD16580 to D.G.M. 


\section{Molecular analyses of early neural pattern decisions in Xenopus. CAREY PHILlIPS (Department of Biology, Bowdoin College, Brunswick, ME 04011).}

Orientation of the Xenopus nervous system results from an interplay between the animal-vegetal axis, established during oogenesis, and intracellular rearrangements, apparently cued by the point of sperm entry. Many subsequent cellular pathway choices are necessarily involved in construction of a nervous system and these choices may be reflected by changes in molecular synthetic patterns. Several molecular probes have been constructed to use in assaying for early determinative events leading to neural differentiation. Epi 1, a monoclonal antibody, is being used as a molecular probe to distinguish between cells proceeding along a neural pathway from cells destined to become epidermis. Using the Epi 1 probe, we have determined that the position of the presumptive neural tissue is approximately, although reversibly, established by at least the third cleavage division. We have also determined that the information necessary for expression of the Epi 1 antigen is associated with the cortex of the uncleaved embryo. The molecular nature of this information is being addressed experimentally. We are now in a position to use Epi 1 and other molecular probes to study how a series of pathway choices might interact to produce the final position of neural structures.

Protein-DNA interactions on the 5 ' non-transcribed spacer of Tetrahymena thermophila rDNA. K. RIEKKI AND R. E. PEARLMAN (Department of Biology, York University, Toronto, Ontario, Canada, M3J 1P3).

The 5' non-transcribed spacer (NTS) of the rDNA of $T$. thermophila contains sequences specifying a number of important functions including bidirectional origin of replication, promotion of RNA polymerase I catalyzed transcription, nucleosome phasing, rDNA copy number control, and topoisomerase I cleavage. We have initiated studies to correlate structure with function and to look at sequence specific proteinDNA interaction in this region.

Various restriction fragments in the $5^{\prime}$ NTS have extremely anomalous mobility when electrophoresed through polyacrylamide gels suggesting sequence directed bending. In the center proximal (650 bp) Taql$X b a I$ fragment, the bending locus appears to be in the 3 ' portion of this fragment. Using crude nuclear protein extracts and an electrophoretic mobility shift assay to assess protein-DNA interaction, we have demonstrated protein-DNA interaction in this Tagl-Xbal fragment. Greater interaction and specificity appears to occur with the $5^{\prime}$ region of this sequence. Binding of protein from the same nuclear extracts also occurs to the $420 \mathrm{bp} \mathrm{Xbal-Xbal} \mathrm{fragment.} \mathrm{Competition} \mathrm{for} \mathrm{this} \mathrm{binding} \mathrm{with} \mathrm{unlabelled} \mathrm{DNA} \mathrm{from} \mathrm{wild}$ type C3 and from the rmm 1 mutation (Larson et al., Cell 47: 229-240 (1986)) suggests interesting and possibly functionally significant specificity of this interaction. DNase I footprint experiments and protein fractionation are in progress in attempts to further assess the specificity of these protein-DNA interactions.

Effect of photoperiod and melatonin in glucosaminidase activity during newt limb regeneration. M. RIVERA, R. APONTE, F. CALIMANO, AND F. VAlles (Cayey University College, Department of Biology, Cayey, P.R. 00633).

The effect of continuous light, continuous darkness, and melatonin on the activity of the lysosomal enzyme $\mathrm{N}$-acetyl glucosaminidase during limb regeneration in the newt Notophthalmus viridescens was studied. Animals exposed to continuous light showed more enzyme activity than the controls during the first 12 days after amputation. In these animals the highest peak of activity was exhibited on day 7 , whereas the controls showed the highest peak at day 5 . The pattern of activity is the same in both groups. In animals exposed to continuous darkness, the highest peak is observed on day 10 after amputation; the activity pattern is delayed as compared with controls. Animals whose forelimbs were amputated and were injected with melatonin showed a decrease in enzyme activity. The highest peak was detected on day 12 after amputation. The relationship between darkness and melatonin is discussed. Part of this work is supported by NIH Grant 5 S14 RR02640. 
Studies on a transplantable monomyelocytic leukemia and in the offspring from crosses between transplanted and normal BALB/c mice. E. RODRIGUEZ, J. MITRA, AND J. LOBUE. (Department of Biology, New York University, New York City, New York).

In vivo cytogenetical studies on a transplantable monomyelocytic leukemia (MML) initially induced in female BALB/c mice (Fredrickson et al., J. Natl. Cancer Inst. 48: 1597-1605, 1972) by the Rauscher leukemia virus (RLV) has revealed: (a) The presence of a marker deleted chromosome 18 in all somatic tissues examined (bone marrow, peripheral blood, and spleen) restricted to female transplanted cells; (b) a high degree of centromeric associations mainly in peripheral blood; and (c) in MML transplanted mice, chromosome \#19 shows presence of NOR in addition to chromosomes 12,15,16, and 18, whereas control animals have NORs on chromosomes $12,15,16$, and 18 .

The disease has been shown to cause abnormalities in meiosis (Segenreich et al., Genetics 113: s20, 1986). Male MML transplanted animals have been mated at mid stage of the disease (third day) with normal females. Analysis of the offspring is presently being performed. Litter size and phenotypical characteristics of the offspring were normal. To date no significant cytogenetical abnormalities have been found in the somatic tissues of the offspring and hematological parameters on peripheral blood seem to be normal.

\section{Localization of laminin and collagen IV transcription in mouse teratocarcinomas and embryos by in situ hybridization. M. B. ROGERS AND L. J. GUDAS (Dana-Farber} Cancer Institute and Department of Biological Chemistry and Molecular Pharmacology, Harvard Medical School, Boston, MA).

Mouse F9 teratocarcinoma cells can be induced in culture to form two distinct extra-embryonic cell types: parietal and visceral endoderm. F9 cells grown in monolayer and treated with retinoic acid (RA) differentiate into parietal endoderm while cells allowed to form aggregates in the presence of RA form embryoid bodies with an outer layer of visceral endoderm. Visceral endoderm is characterized by the synthesis of alpha-fetoprotein and the basement membrane components laminin and collagen IV. The transcription of these genes has been characterized by in situ hybridization of radioactive recombinant probes to frozen sections of embryoid bodies. In contrast to previous observations from other laboratories on basement membrane protein expression, laminin and collagen IV mRNAs were found in the outer cell layer of embryoid bodies whether or not RA was present. Additional experiments are extending the technique to sections of preimplantion mouse embryos.

Meiotic chromosome studies in BALB/c mice bearing a transplantable mono-myelocytic leukemia (MML) and in the offspring of normal females crossed with transplanted males. E. J. SEGENREICH, J. MITRA, AND J. LOBUE (Department of Biology, New York University, New York, NY).

Meiotic chromosome studies have been performed on BALB/c mice transplanted with mono-myelocytic leukemia (MML), an acute disease induced by a virally (RLV) transformed cell (Fredrickson et al., J. Natl. Cancer Inst. 48: 1597-1605, 1972). Results indicate that MML transplantation is associated with increases in meiotic chromosomal aberrations. Levels of aneuploidy, polyploidy, structural alterations, and precocious separations rise throughout the six day disease. Spermatogonial chromosomes also show increases in aneuploidy, polyploidy, and structural alterations. These mitotic chromosomes exhibit a higher degree of anomalies than those in other tissues of the leukemic animal (E. Rodriguez, unpub. data). Successful mating of normal females and mid-stage (day 3) MML transplanted males has produced phenotypically and, as of one year of age, hematologically normal litters. Preliminary studies of the offspring have revealed mostly insignificant changes in the frequencies of meiotic aberrations compared to the control litters. Only a slight increase in polyploidy is observed. Spermatogonial mitosis also shows slight rises in the level of polyploidy along with centromeric affinity. The nature of susceptability to the pathological parameters of the disease as well as to meiotic disturbances, will be determined by MML transplantation into the offspring. 
Spatial patterning of neuronal differentiation in the leech. M. SHANKLAND AND M. Q. MARTINDALE (Department of Anatomy and Cellular Biology, Harvard Medical School, Boston, MA).

We have examined the spatial patterning of neuronal differentiation in the metameric nerve cord of the leech using an antibody to molluscan small cardioactive peptide B (SCP). In the adult, anti-SCP stains an unpaired interneuron that is present only on the right or left side of the segmental ganglion in abdominal segments 1-3 and 18-21. Both antibody staining and intracellular injection of Lucifer Yellow reveal that this neuron extends an axon through the connective nerve into adjacent ganglia. The unpaired neuron is equally likely to lie on either side of a given segment, but there is greater than $95 \%$ likelihood that it will be on alternate sides (e.g., right:left) in successive segments. In the embryo, every abdominal ganglion initially has a bilateral pair of these neurons and both express SCP-like immunoreactivity. As development proceeds one member of the pair ceases to stain in segments 1-3 and 18-21, while both members of the pair cease to stain in segments 4-17. The right:left alternation observed in the mature pattern could arise because the unpaired neuron in one ganglion influences the right:left decision in adjacent ganglia through its interganglionic axon. We have obtained support for this idea by showing that the pattern of alternation is locally disrupted by transecting the embryonic nerve cord prior to the onset of asymmetry.

Atrial natriuretic peptide (ANP) levels during rat ontogeny. R. E. SICARD AND J. C. WERNER (Division of Pediatric Cardiology, Department of Pediatrics, Rhode Island Hospital, \& Program in Medicine, Brown University, Providence, RI).

ANP is an important modulator of fluid/electrolyte and pressure homeostasis. Its role and mechanisms of action have been extensively explored in adults; however, adequate appreciation of the developmental physiology of ANP is limited. Accordingly, we have begun exploring ANP metabolism during rat development. Levels of immunoreactive ANP (ir-ANP) were determined by radioimmunoassay (reagents from Amersham) in amniotic fluids or plasma during the last trimester of pregnancy and the first 2 months of life. During the last trimester, amniotic fluids displayed a biphasic change in ir-ANP levels: rising from $<10 \mathrm{fmol} / \mathrm{ml}$ ( 13 days gestation) to $31.4 \pm 7.3 \mathrm{fmol} / \mathrm{ml}(\mathrm{n}=29 ; 18-19$ days gestation), then falling to $<10$ $\mathrm{fmol} / \mathrm{ml}(\mathrm{n}=5)$. Plasma concentrations of ir-ANP remained $<10 \mathrm{fmol} / \mathrm{ml}$ during this time, then rose during the first month $[25.9 \pm 7.1 \mathrm{fmol} / \mathrm{ml}, \mathrm{n}=21]$ to approximately adult levels $[22.3 \pm 5.4 \mathrm{fmol} / \mathrm{ml}, \mathrm{n}$ $=29$ ]. These data suggest that significant changes in ANP metabolism and activity might be occurring during the perinatal period in rats.

Localization of a gene product in Drosophila that influences biological rhythms.

K. K. SiWICKI, C. EASTMAN, M. RoSBaSH, AND J. C. Hall (Biology Department, Brandeis University Waltham, MA 02254).

The period (per) gene of Drosophila melanogaster influences the period of circadian rhythms as well as an ultradian rhythm (period ca. $1 \mathrm{~min}$ ) in the fly's courtship song. To begin to investigate how the gene regulates these complex behaviors, we used an antibody specific for the per protein to examine its anatomical distribution with immunocytochemical methods. Staining was detected in the eyes, optic lobes, and brain of wild type flies, but was not present in per mutants. Rhythmic fluctuations in the intensity of the staining were apparent: the pattern was much more prominent at night than during the day. The day/night cycling was observed during entrainment ( $12 \mathrm{~h}$ light/12 h dark) and continued to cycle in constant darkness, suggesting that an endogenous circadian oscillator may regulate the expression of the per gene, which itself influences circadian rhythms. Supported by NS-07873 to K.K.S. and GM-33205 to M.R. and J.C.H.

Centrosomes in echinoderm development. G. SLUDER, F. J. MILLER, K. LEWIS, AND C. L. REIDER (Worcester Foundation for Experimental Biology, Shrewsbury, MA 01545).

In animal cells, centrosomes are an ensemble of poorly understood organelles found at the ends of the mitotic or meiotic spindles. Centrosomes are required for the establishment of the bipolar spindle axis, chromosome orientation/alignment, chromosome movement, and the establishment of the cleavage furrow. We will briefly introduce the centrosome and then discuss the origin of the centrosomes used in the development of the echinoderm zygote. We demonstrate for sea urchin eggs that the centrosomes used in development come only from the sperm; the egg centrosome is apparently lost. We then describe our 
characterization of centrosome inheritance in starfish eggs. This system is interesting because the egg is fertilized at miosis I when it contains two functional centrosomes. However, only the male centrosome is used in development; the female centrosomes are functionally lost prior to first mitosis. We will describe ongoing experiments which seek to: (a) provide an understanding of what functional and structural aspects of the female centrosomes are lost; (b) show that male and female centrosomes have intrinsically different stabilities; (c) determine when, during the meiotic sequence, the female centrosomes are destabilized.

Mouse cellular retinoic acid binding protein: cloning, $c D N A$ sequence and $m R N A$ expression during the retinoic acid-induced differentiation of $F 9$ wild type and $R A$ 3-10 mutant teratocarcinoma cells. CAROL M. STONER AND LORRAINE J. GUDAS (Dana-Farber Cancer Institute and Dept. of Pharmacology, Harvard Medical School, Boston, MA).

Retinoic acid, a natural derivative of vitamin A (retinol), induces mouse F9 teratocarcinoma stem cells to differentiate into nontumorigenic parietal endoderm cells. The mouse cellular retinoic acid binding protein (CRABP) has been implicated in the mechanism of action of retinoic acid (RA). A mutant F9 teratocarcinoma cell line, RA-3-10, which possesses less than 5\% of the wild type level of CRABP activity, fails to differentiate in response to RA. To study the role that CRABP plays in the RA-induced differentiation process, we cloned the mouse CRABP cDNA and determined its full-length sequence. Southern analysis of F9 genomic DNA hybridized to CRABP cDNA suggests that the CRABP gene is present as a single copy gene in the haploid genome and that the gene contains two introns. Northern analysis shows that the CRABP mRNA is a single, low abundant mRNA approximately 800 bases in length. The steady state CRABP mRNA level remains relatively constant during the RA-induced differentiation of F9 wild type cells. The CRABP mRNA level is approximately 20-fold lower in the RA-3-10 mutant stem cells than in F9 wild type stem cells.

Cyclins and the cell cycle of early clam embryos. K. I. SWENSON ${ }^{1}$ AND J. V. RUDERMAN $^{2}$ ('Dept. of Anatomy and Cellular Biology, Harvard Medical School, Boston, MA; ${ }^{2}$ Dept. of Zoology, Duke University, Durham, NC).

Fertilized oocytes of the surf clam require new protein synthesis in order to complete meiosis. New protein synthesis is also required during each cell cycle of the early embryo for the completion of the mitotic divisions as well (Hunt and Ruderman, unpub.). Possible candidates for these $\mathbf{M}$-phase inducing proteins are the cyclins $A$ and $B$, the levels of which periodically accumulate and disappear as a function of the mitotic cell cycle (Evans et al., 1983. Cell 37: 389; Swenson et al., 1986. Cell 47: 861; Westendorf, unpub.). The suspected $M$-phase inducing role of cyclin A has been confirmed by showing that Xenopus oocytes, when microinjected with in vitro synthesized SP6 cyclin A mRNA, enter meiosis. The activity of cyclin B in this assay system is unknown. Recently we found that cyclin A was tightly associated with a kinase activity. We are interested in the functional properties and specificities of this kinase activity and its involvement, if any, in the ability of cyclin $A$ to induce $M$-phase.

Molecular genetics of early embryogenesis in C. elegans. A. TELFER, D. LEVITAN, U. GiAMBARELLA, AND D. T. STINCHCOMB (Harvard University, Cambridge, MA).

The first division of embryogenesis in the nematode, Caenorhabditis elegans, produces blastomeres that display different cell division patterns and are determined to express different developmental potentials. Mutations in one class of maternal effect genes disrupt early development: the first division produces blastomeres that divide synchronously and fail to express certain differentiated cell types. These mutants are termed par for they are defective in the partitioning of germ line granules, they fail to segregate factors that control cell cycle rates, and they possibly fail to partition determinants of some cell types. We have identified and isolated restriction fragment polymorphisms (RFLPs) on either side of the par-1 locus. DNA between the two markers has been cloned. We will delimit the par-1 gene by identifying maternal transcripts and by microinjecting cloned DNA to assess its function. Similarly, a RFLP in or near the par-2 gene has been identified and adjacent DNA has been isolated. Characterization of the par gene products may help us understand how early blastomeres become determined during development. 


\section{Retinoids and pattern formation in vertebrate limbs. CHRISTINA THALLER AND GREGOR EICHELE (Dept. of Physiology, Harvard Medical School, Boston, MA).}

All-trans-retinoic acid induces striking digit pattern duplications when locally applied to the developing chick limb bud. Instead of the normal digit pattern (234) a mirror-symmetrical 432234 pattern can be specified. Hence, retinoic acid closely mimics posterior limb bud tissue (known as the zone of polarizing activity, ZPA) that causes very similar duplications when grafted to an anterior site of a host limb bud. This raises an intriguing possibility: that retinoic acid is a natural signalling substance involved in limb pattern formation. We discovered that chick limb buds contain endogenous retinoic acid and found that retinoic acid but not its biologically inactive precursor retinol, forms a concentration gradient across the limb anlage with a highpoint in the posterior domain of the limb bud, the part that contains the ZPA. Moreover, the amount of endogenous retinoic acid detected in the limb bud is the same as required to induce duplications when retinoic acid is provided exogenously. To examine whether retinoic acid is produced in situ, we locally applied its precursors, all-trans-retinol and all-trans-retinal, in radioactive form. Both compounds are metabolized: retinol to retinal and retinal to retinoic acid. These observations strengthen the hypothesis that retinoic acid is a local chemical mediator involved in the specification of the anteroposterior limb pattern.

Region specific expression of mouse homeo box genes. M. F. UTSET ${ }^{1}$ A. AWGULEWITSCH $^{2}$, W. MCGINNIS ${ }^{3}$, AND F. H. RUDDLE ${ }^{1,2}$ (Departments of ${ }^{1}$ Human Genetics, ${ }^{2}$ Biology, and ${ }^{3}$ Molecular Biophysics and Biochemistry, Yale University, P.O. Box 6666, New Haven, CT 06510).

Mammalian homeo box genes show extensive homology to Drosophila homeotic and segmentation genes. As a step toward determining their functions, we are studying the patterns of homeo box gene expression during mouse development. Results from a number of laboratories indicate that several mouse homeo box genes display region specific patterns of expression within the developing central nervous system and mesoderm. For example, Hox-2.1 expression within the central nervous system of the newborn mouse and the 13.5 day mouse embryo appears restricted to the medulla and spinal cord, whereas at the same stages Hox-3.1 expression is found within the spinal cord posterior to the third cervical vertebra (Science 235: 1379-1382). At earlier embryonic stages Hox-3.1 expression is also detected in a spatially restricted pattern in mesodermal tissues. These patterns of expression are consistent with the hypothesis that mouse homeo box genes perform region specific developmental functions akin to those of Drosophila homeotic genes.

Visualization of actin with rhodamine phalloidin in the zebrafish egg. J. S. WOLENSKI AND N. H. HART (Department of Biological Sciences, Rutgers University, New Brunswick, NJ).

The distribution of polymerized actin in zebrafish (Brachydanio) eggs was determined using rhodamine phalloidin and fluorescence microscopy. Unactivated eggs exhibited a prominent and continuous band of fluorescence just beneath the plasma membrane. Whole eggs showed little evidence of staining in the remaining cortical cytoplasm. However, staining was visible around individual cortical granules in cortical fragments prepared from formaldehyde-fixed cells. A prominent, narrow band of staining was also detected at the periphery of activated eggs. Continuity of this fluorescent layer was interrupted at sites of fusion between the egg plasma membrane and exocytosing cortical granules. Gaps in the fluorescence were also evident in eggs treated with cytochalasin B. Our results suggest that polymerized actin is present in cortices of eggs including the site of sperm entry.

Developmental regulation of three testis-specific genes during mouse spermatogenesis. P. C. YeliCK, Y. KWON, P. A. BOWER, K. C. KLEENE, AND N. B. HECHT (Department of Biology, Tufts University, Medford, MA 02155).

Mouse spermatogenesis is the continuous process of development in which a progenitor germ cell differentiates into mature spermatozoa. We have identified three testis-specific genes, mouse protamine 1 (mP1), mouse protamine 2 (mP2), and mouse transition protein 1 (mTP1), all of which are expressed in the haploid phases of spermatogenesis. All three gene products are involved in the nuclear condensation events which occur during the sequential replacement of the nucleosomal histones by mouse protamines. 
All three genes are very similarly regulated. $\mathrm{mP1}, \mathrm{mP2}$, and $\mathrm{mTP} 1$ genes are first transcribed in the round spermatid stage of spermiogenesis. All three mRNAs are translationally regulated in that they are first transcribed and then stored for up to eight days before they are translated. The translated forms of all three mRNAs, as present on polysomes, differ from the RNP-complexed forms in that the poly (A)+ tails are shortened by about 140 nucleotides. We also demonstrate the presence in rat and hamster of gene sequences and testis-specific mRNAs homologous to mP1 and mP2 cDNAs. Comparison of protamine 1 and 2 gene expression in rat, hamster, and mouse demonstrates variable expression of the protamine 1 and 2 genes that is also reflected in the protamine 1 and 2 content of hamster, rat, and mouse sperm. 